\title{
EDITORIAL
}

\section{Skill training in medical practice}

\section{By}

\section{Raouf Sallam}

\begin{abstract}
I am sure most of us have tried to thread a needle and failed in his first,and may be second and third,attempt and then succeeded. Very few succeeded from the first time and fewer still failed repeatedly and gave up. This is the nature of technical manual skills that need hand-eye coordination and psychomotor activity. The human being is as such created.
\end{abstract}

When it comes to operative surgery, the technical manual skills are many, much more complex than threading a needle and quite varied. More importantly is that when you are trusted with a human body you are expected to do it right all the time,every time and from the first time. So how was-and still is-surgery being practiced?

From the dawn of history surgery was being practiced,the surgeon to be learned-and then practiced all the skills and tricks of the business by apprenticeship.

That is following,watching and assisting a senior until considered fit enough to operate on his own and maybe teach others. So the training in surgery was by years of watching and assisting before operating. The famous dictum of "see one,do one,teach one "is an over compression of the real process. Ideally the trainee must watch some,assist in many,be assisted by a senior in some,do some under direct supervision, do some with indirect supervision and then operate on his own with his results being monitored.

This time honored training pattern was challenged in the last three decades by certain changes in surgical practice. The changes may be unrelated but they happened at almost the same time:

- For managerial reasons the training period of working under supervision was reduced or eliminated.

- The working hours of surgeons and trainees were reduced after noting that most of their mistakes occur in the last few hours of a long shift.

- Junior consultants were noted to need few months or years to reach the plateau of their expertise. Surgeons admit that their mistakes were "in the first few cases". Which in a sense means that they complete their learning curve on the patients. That is something unacceptable by either today's surgeons or today's public.

- The introduction of new invasive techniques in many specialities where their were no seniors trained to perform them, let alone teach them to others in the traditional way.

All the above factors made it necessary to look for training outside the OR "operating room". That is training on the skill before applying it to the patient. Simply, training then operating.

Training outside the OR took many forms:

- training on cadavers..........obstructed by ethical concerns.

- training on inanimate objects...........suitable only for limited skills.

- training on animal tissue............suitable only for limited skills.

- training on anesthetized animals.........objected by the strong animal lovers lobby in the west.

- virtual reality training: the introduction of MIS " minimally invasive surgery" that depends wholly or mainly on camera,video and screen has quickly prompted the idea of creating software for training on virtual reality where you do not need any material tissue for training

Experience with laparoscopic cholecystectomy, a video assisted camera and screen dependent procedure ,revealed two serious shortcomings:

- the two dimensional screen vision may result in visual illusions. The problem with the visual illusion is not that you do not see well,on the contrary you see very well but what you see is not what is there.Simply what you see is not what you are looking at - an almost complete lack of haptics " touch feed back", since you can feel the tissues only through long thin instruments reaching the tissues rather indirectly and incompletely 
Of our human five senses we only use two in surgical procedures steps, vision and touch .And if these are affected that is a great handicap.so far,those operating through the screen are surgeons with a lot of experience in open surgery that compensated for this handicap. Such surgeons are gradually getting fewer and fewer.

Luckily, the industry quickly listened to the surgeons concerns and began to include 3D vision and a haptic component to the newer models of the video assisted surgery equipment. These are not perfect yet but promising to soon be as close as possible to the natural see and touch of the human tissue. The same is applied to the training simulators software which also began to include situations like diathermy smoke, aberrant anatomy etc. such software can also count the mistakes done by the trainee and give a final evaluation. Studies showed that simulation training helped the trainee to perform the skill in a shorter time and with less errors, but the extent of actual benefit to the patient is not yet clear.

When I used surgery as an example of the training /practicing issue in medicine, I didn't mean surgery as a speciality, but I meant surgery as an invasive technique in procedures applied to the patient. Maybe I used the word surgery being the oldest speciality in the profession that uses invasive techniques as its main method of treatment. But now almost all specialities in modern medicine uses invasive techniques in their practice involving some surgical component and many video assisted steps. Consider interventional radiology, invasive cardiology, therapeutic endoscopy and others.

In a recent surgical conference in Cairo, Dr.Morad Sallam of Cairo university and St'Thomas hospital of England,who is involved in post-graduate training, drew our attention, in one of his presentations, to this change in the pattern of training. He called it a change of paradigm and I dare call it a revolution. It is a radical change from a centuries long type of training, a change that will last. Where do we,here in Egypt,stand from all of this? Simulation training will soon be a requirement for getting the privilege of operating independently. Even if this is not applied in Egypt,it will still face our Egyptian doctors who wish to work abroad and our hospitals seeking international accreditation. Besides, if it is something good for the patient we should apply it. The question arises...can we?

Virtual reality simulators with 3D vision and a haptic component and including unusual or rare operative situations ,needed for a complete training,are very expensive specially when we need to cover all the skills and procedures of different branches in medicine and need to train most of the junior staff. So we will have to rely more on inanimate simulators "dry box", and simulation training on animal tissue and anesthetised animals. And we will have to rely less on virtual reality simulators which,though more aristocratic and less messy, are also less natural and less affordable.

We have,here in Egypt,the advantage of a culture that permits us to use animals for research and training as long as the animal does not endure pain or suffer in any way or get separated from its offspring. In the early 1990's we had very good results from training on different kinds of laparoscopic procedures by using anesthetised pigs in the animal lab. of our faculty here. We also used fresh animal tissue for training on isolated skills e.g.intestinal anastomoses.

We all know that simulation training will not be replacing apprenticeship but will just be augmenting it,trying to fill in the gap in apprenticeship training. But having said all this I still have a question and a worry:

The question: is there any evidence that simulation training,when augmenting apprenticeship training, will get the trainee to the plateau of competence required for the safe performance and thus accommodate the hurtful part of the learning curve away from the patient? Or will we still need the patient for part of the learning curve and again face the problem of the vulnerability of the first few patients in a novice surgeon's career?

The worry: We know that the ultimate in skill training, regardless the nature of the skill, is for the trainee to reach the level of automatism, that is performing the skill automatically as if without thinking, like a lady knitting tricot. And I wonder if it is advisable to reach this level in performing surgery. I believe a surgeon should be thinking all the time and not working automatically .We know that the commonest cause of errors in surgery is lack of concentration, which is a constant companion of automatism.

We know that occasionally senior surgeons, after years on the plateau of competence do silly mistakes. We usually attribute this to over confidence. I attribute it to automatism. May be over confidence has sneakily tempted him to automatism.

A surgeon should remember that every time he is operating, he is operating on a different patient, human tissues may be similar but not identical. There are no standard specifications for the human tissues and certainly no typical situations.

Every step in surgery should be custom made to the patient under your hands.

Dear colleague "beware of automatism in surgery, surgery is no field for automatism ".

Prof. Raouf Sallam F.R.C.S.

Editor-in-Chief 